\title{
EFEITO DO CARREGAMENTO CÍCLICO SOBRE AS PROPRIEDADES DE FLEXÃO DE PAINÉIS COMERCIAIS DE MDF E MDP
}

\author{
Cláudio Henrique Soares Del Menezzi ${ }^{1}$, Cristiane Moreira Tavares dos Santos², Joana Mendes Ferraz², \\ Sabrina Andrade Martins ${ }^{3}$, Rafael Rodolfo de $\mathrm{Melo}^{4}$, Milton Luiz Siqueira ${ }^{5}$, Valeria Maria de Figueiredo Pazetto
}

(recebido: 17 de janeiro de 2010; aceito: 27 de maio de 2011)

RESUMO: Conduziu-se este trabalho, com o objetivo de avaliar o efeito fadiga por meio de diferentes ciclos de carregamento (20.000, 40.000 e 80.000 ciclos) na deformação e nas propriedades de flexão estática de painéis comerciais de medium density fiberboard (MDF) e medium density particleboard (MDP). Cada ciclo de carga teve a duração de 2,2 segundos $(0,45 \mathrm{~Hz})$ e a carga aplicada foi correspondente a $25 \%$ do módulo de ruptura de cada painel. Após os ensaios, foi avaliada a deformação com (final) e sem carga (residual), e realizados ensaios de flexão estática para determinação das propriedades de flexão dos painéis. De acordo com os resultados, não houve efeito deletério nas propriedades de flexão para ambos os tipos de painel para os diferentes ciclos de carregamento. Entretanto, foi identificado o efeito dos ciclos de carregamento na deformação residual dos painéis MDF.

Palavras-chave: Painéis reconstituídos, carregamentos cíclicos, fadiga.

\section{CYCLIC LOADING EFFECT ON THE FLEXURAL PROPERTIES OF COMMERCIAL MDF AND PARTICLEBOARD PANELS}

\begin{abstract}
This paper aimed at evaluating the effect of fatigue through different loading cycles (20,0000; 40,000 and 80,000 cycles) on deflection and flexural properties of commercial MDF and particleboard panels. A 2.2 seconds (0.45 Hz) cyclic loading was employed and the applied load about $25 \%$ of modulus of rupture was used. After the tests, final deflection (with load), residual deflection (without load) and flexural properties of the panels were evaluated. According to the results, the flexural properties for both kinds of panels were not affected by the loading cycles tested. However, it was identified that the MDF residual deflection was affected between 20,000 and 80,000 loading cycles.
\end{abstract}

Key words: Reconstituted panels, cyclic loading, fatigue,

\section{INTRODUÇÃO}

Os compostos à base de madeira apresentam diversas aplicações e usos, seja como elementos estruturais, tais como: pisos, paredes e vigas, dentre outros elementos em complexos residenciais, ou não estruturais, os quais são empregados principalmente na indústria de móveis (THOMPSON et al., 2005). No Brasil, destacamse os usos de compostos não estruturais, principalmente na fabricação de móveis.

Para aplicações como elemento estrutural, Sugimoto et al. (2006) destacaram que esses compostos são frequentemente submetidos a carregamentos prolongados, além de serem expostos a ações de intempéries. Esses aspectos proporcionam o surgimento de fadiga, que reduz a capacidade das peças suportarem carga, e consequentemente sua vida útil em serviço. No entanto, Espinosa e Calil Junior (2000) mencionaram que mesmo para usos não estruturais, de medium density fiberboard (MDF) e aglomerados convencionais, também podem sofrer com a ocorrência desse fenômeno.

Fadiga pode ser definida como um processo de mudança estrutural permanente, localizada e progressiva que ocorre em materiais sujeitos às tensões e deformações

\footnotetext{
${ }^{1}$ Engenheiro Florestal, Professor Dr. em Engenharia Florestal - Departamento de Engenharia Florestal - Faculdade de Tecnologia - Universidade de Brasília - Cx. P. 04357 - 70904-970 - Brasília, DF - cmenezzi@unb.br

${ }^{2}$ Engenheira Florestal, Mestre em Ciências Florestais - Departamento de Engenharia Florestal - Faculdade de Tecnologia - Universidade de Brasília Cx. P. 04357 -70904-970 - Brasília, DF - crismtsantos@yahoo.com.br, joanamendesferraz@gmail.com

${ }^{3}$ Bacharel em Tecnologia da Madeira, Doutoranda em Ciências Florestais - Programa de Pós-Graduação em Ciências Florestais - Departamento de Engenharia Florestal - Faculdade de Tecnologia - Universidade de Brasília - Cx. P. 04357 - 70904-970 - Brasília, DF - sabrinaand@gmail.com ${ }^{4}$ Engenheiro Florestal, Doutorando em Ciências Florestais - Programa de Pós-Graduação em Ciências Florestais - Departamento de Engenharia Florestal - Faculdade de Tecnologia - Universidade de Brasília - Cx. P. 04357 - 70904-970 - Brasília, DF - rrmelo2@yahoo.com.br ${ }^{5}$ Engenheiro Mecânico, Professor Dr. em Engenharia Mecânica - Departamento de Engenharia Mecânica - Faculdade de Tecnologia - Universidade de Brasília - 70919-970 - Brasília, DF - milton@unb.br

${ }^{6}$ Desenhista Industrial, MS em Ciências Florestais - Laboratório de Ensaio de Móveis - Departamento de Engenharia Mecânica - Universidade de Brasília - 70919-970 - Brasília, DF - valeriapazetto@gmail.com
}

Cerne, Lavras, v. 17, n. 3, p. 403-409, jul./set. 2011 
flutuantes ou não, podendo culminar em rachaduras, ou em completa ruptura após um determinado tempo ou suficiente número de flutuações. A falha por fadiga em materiais resulta da aplicação cíclica ou estática de uma tensão menor que o valor necessário para causar um comportamento inelástico ou uma fratura sob condições de carregamento monotônico (SMITH et al., 2003). Esse fenômeno está relacionado com o comportamento reológico da madeira e dependendo da magnitude e da velocidade da aplicação de carga, a resistência mecânica pode ser mais ou menos comprometida (BODIG; JAYNE, 1993).

Conforme mencionado por Melo e Del Menezzi (2010), a madeira ou compostos de madeira quando submetidos a carregamentos permanentes, cíclicos ou não, mesmo dentro do limite de proporcionalidade, após a suspensão da aplicação de carga podem apresentar uma deformação residual e irreversível. Esse aspecto faz com que a capacidade do material de suportar carga seja reduzida ao longo do tempo. Apesar disso, na maioria dos estudos em que se busca avaliar a resistência da madeira e derivados, tem sido dado maior ênfase à resistência sob carga estática, e relativamente pouca atenção para os efeitos causados cargas cíclicas.

Bao et al. (1996), estudando a resistência à fadiga de painéis MDF, aglomerado, OSB e compensado, observaram que esses materiais podem suportar ciclos de carregamento extremamente altos $\left(>10^{6}\right)$, quando o nível de tensão aplicado equivale à $30 \%$ do módulo de ruptura (MOR). Um rápido decréscimo no tempo para ocorrer a fadiga foi observado para todos os materiais, quando o nível de tensão aplicado aumentou para 50\% do MOR. Em um nível de tensão de $70 \%$ do MOR, a duração da fadiga decresce de um nível de 400.000 para 10.000 ciclos. Uma forte relação não linear expressa de forma adequada à relação entre o nível de tensão aplicada e tempo para ocorrer a fadiga (BAO et al., 1996).

Bao e Eckelman (1995), estudando a deformação e a fadiga de painéis MDF, MDP e OSB utilizados para a produção de móveis, observaram que, quando o nível de tensão aplicado é de $70 \%$ do MOR o número de ciclos decresce para 405 ciclos para o MDF e 10.887 ciclos para o OSB, e, com isso, verificaram que, quando o nível de tensão aplicada aumenta, o tempo ocorrência da fadiga decresce. As falhas em painéis MDF começam a aparecer quando os níveis de tensão alcançaram $40 \%$ do MOR.

De acordo com Pritchard et al. (2001), as normas americanas estabelecem que um item para utilização em mobiliário deva resistir a 200.000 ciclos antes de falhar.
A tensão aceitável para os ensaios para satisfazer as normas é em torno de $40 \%$ do MOR, para MDF, OSB e Chipboard. Segundo os mesmos autores, alguns dos estudos recentes relatam que a umidade relativa e a temperatura afetam as propriedades mecânicas dos painéis, no entanto, quando a umidade relativa do ar varia de $30 \%$ a $65 \%$ essa tem pouco efeito na fluência dos chipboards sujeitos à fadiga cíclica.

Segundo Smith et al. (2003), muita atenção tem sido dada para estudos de falha em madeira causada por carga estática e relativamente pouca atenção para os efeitos da carga cíclica. Diante do exposto, objetivou-se, no presente estudo, avaliar o efeito da fadiga causada por diferentes números de ciclos de carregamento (20.000, 40.000 e 80.000 ciclos) na deformação e nas propriedades de flexão estática de painéis comerciais de MDF e MDP.

\section{MATERIAL E MÉTODOS}

\subsection{Painéis de madeira}

Foram utilizados painéis comerciais de MDF (medium density fiberboard) e MDP (medium density particleboard) adquiridos no mercado local. O MDF apresentava-se com dimensões 1830 × $2750 \mathrm{~mm}$ e espessura de $15 \mathrm{~mm}-\mathrm{e}$, conforme o fabricante, foi produzido a partir de Pinus sp. e resina à base de uréia-formaldeído - com densidade variando entre 650 a $700 \mathrm{~kg} / \mathrm{m}^{3}$. O painel de MDP apresentava-se com dimensões 1830 × $2750 \mathrm{~mm}$ e espessura de $15 \mathrm{~mm}$, e conforme o fabricante foi produzido a partir de Eucalyptus sp. e resina à base de uréia-formaldeído - com densidade variando entre 550 e $600 \mathrm{~kg} / \mathrm{m}^{3}$. Informações adicionais sobre os materiais e a obtenção dos corpos-deprova podem ser obtidas em Pazetto (2009).

\subsection{Ensaio de fadiga}

Para os ensaios foram retiradas nove amostras com $400 \mathrm{~mm}$ de comprimento, $76 \mathrm{~mm}$ de largura e $15 \mathrm{~mm}$ de espessura - de cada painel, totalizando 18 amostras. O número e a duração de cada ciclo foram estabelecidos por um controlador lógico programável (CLP). O controle da carga aplicada foi feito com o auxilio de um manômetro digital. Para os apoios e o atuador de carga, seguiu-se o padrão estabelecido para o ensaio de flexão estática, estabelecido segundo ASTM D - 1037 (AMERICAN SOCIETY FOR TESTING AND MATERIALS - ASTM, 1999).

Os ensaios de fadiga nos painéis MDF e MDP (Figura 1) foram feitos em uma máquina universal de ensaios com sistema de funcionamento pneumático no Laboratório de Ensaios em Móveis (LabMov) da Universidade de Brasília, laboratório acreditado pelo

Cerne, Lavras, v. 17, n. 3, p. 403-409, jul./set. 2011 

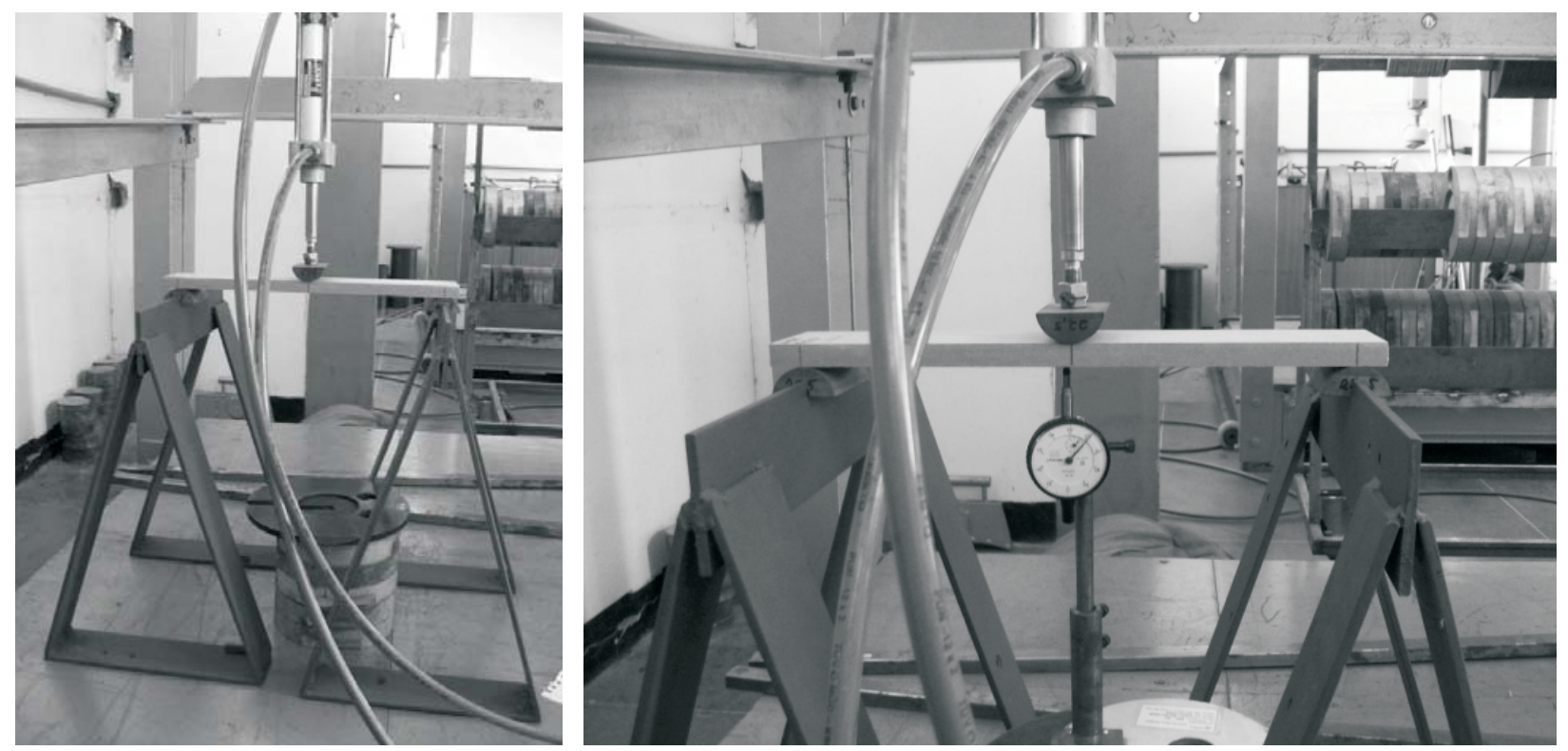

Figura 1 - Ensaio de fadiga e medição da deformação.

Figure 1 - Fatigue assay and deformation measurement.

INMETRO para aferição da qualidade de móveis. As condições de temperatura e umidade no laboratório foram monitoradas durante os ensaios, apresentando os valores de $25 \pm 2^{\circ} \mathrm{C}$ e $60 \pm 5 \%$ respectivamente.

A carga do atuador foi aplicada em L/2 (flexão estática de três pontos) e foi definida para se produzir uma tensão nas bordas equivalente a $25 \%$ do MOR dos painéis, conforme obtido por Pazetto (2009). Assim, a carga do atuador foi regulada para aplicar $270 \mathrm{~N}$ em cada ciclo para o MDF e 120N para o MDP. Segundo Pazetto (2009), a carga média no limite proporcional desse material variou de $628-657 \mathrm{~N}$ para o MDF e de $363-412 \mathrm{~N}$ para o MDP. Desse modo, a cada ciclo de carga o painel era solicitado dentro de seu regime elástico. Os corpos-de-prova foram ensaiados segundo três ciclos de carregamento: 20.000, 40.000 e 80.000 ciclos (Tabela 1).

Tabela 1 - Número de amostras para cada ciclo de carregamento. Table 1 - Number of samples for each loading cycle.

\begin{tabular}{lcccc}
\hline & \multicolumn{3}{c}{ Número de ciclos } & \multirow{2}{*}{ Carga } \\
\cline { 2 - 4 } Tipo de painel & 20.000 & 40.000 & 80.000 & $(\mathrm{~N})$ \\
& $(12,2 \mathrm{~h})^{\mathrm{a}}$ & $(24,4 \mathrm{~h})$ & $(48,8 \mathrm{~h})$ & \\
\hline MDF & 3 & 3 & 3 & 270 \\
MDP & 3 & 3 & 3 & 120 \\
\hline
\end{tabular}

Nota: a: duração do ensaio.
A forma do ciclo escolhida para os ensaios foi o quadrado real (actual square), em que o maior tempo do ciclo a carga permanece residente na amostra com a máxima tensão (SMITH et al., 2003). Cada ciclo tinha a duração de 2,2 segundos - 2 segundos de duração da carga e 0,2 segundo de tempo de aplicação e retorno do atuador, correspondendo, assim, a uma frequência de 0,45 $\mathrm{Hz}$ (Figura 2).

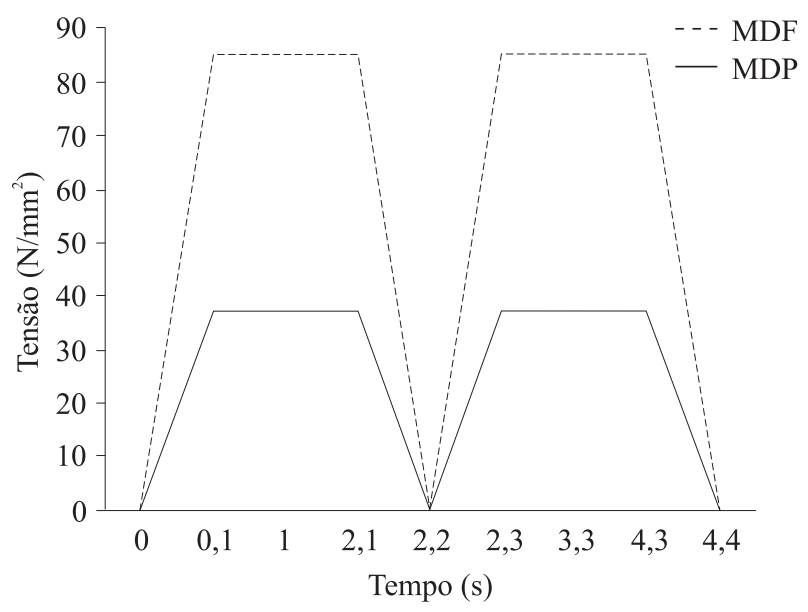

Figura 2 - Forma e duração dos ciclos de carregamento.

Figure 2 - Shape and duration of loading cycle.

Cerne, Lavras, v. 17, n. 3, p. 403-409, jul./set. 2011 
Para medir a deformação final $\left(\varepsilon_{\text {final }}\right)$ e residual $\left(\varepsilon_{\text {residual }}\right)$, utilizou-se um relógio comparador (Mitutoyo) de precisão de $0,01 \mathrm{~mm}$ (Figura 1). As medições foram feitas após o ensaio de fadiga, onde se determinou a deformação dos corpos-de-prova com carga $\left(\varepsilon_{\text {final }}\right)$ e sem carga $\left(\varepsilon_{\text {residual }}\right)$. Após o ensaio de fadiga, os painéis foram ensaiados em flexão estática até a ruptura segundo os procedimentos da norma ASTM D - 1037 (ASTM, 1999) para determinação do MOE e MOR.

\subsection{Análise dos resultados}

Para se avaliar o efeito dos diferentes níveis de carregamento cíclico sobre as deformações e as propriedades de flexão dos painéis MDP e MDF foi rodada inicialmente uma análise da variância (ANOVA). Quando a ANOVA foi significativa, foi feita uma posterior comparação das médias dos três tratamentos (20.000, 40.000 e 80.000 ciclos) por meio do teste de Tukey HSD ao nível de $\alpha=0,05$.

\section{RESULTADOS E DISCUSSÃO}

Para as deformações avaliadas pode-se observar que o número de ciclos afetou significativamente apenas a deformação residual $\left(\varepsilon_{\text {residual }}\right)$ dos painéis MDF, não sendo observado efeito significativo para as demais deformações avaliadas. O número de número de ciclos também não afetou significativamente as propriedades de flexão para ambos os tipos de painéis avaliados (Tabela 2), o que indica que seriam necessários valores superiores a 80.000 ciclos para promover a redução de resistência das amostras.

Tabela 2 - Sumário da ANOVA para o efeito do número de ciclos sobre as propriedades de painéis MDF e MDP.

Table 2 - ANOVA summary for the effect of cyclic loading on $M D F$ and MDP panel properties.

\begin{tabular}{lccc}
\hline Tipo de painel & Propriedade & Fcalc. & Sig. \\
\hline \multirow{3}{*}{ MDF } & $\varepsilon_{\text {final }}$ & 2,842 & $0,135^{\mathrm{ns}}$ \\
& $\varepsilon_{\text {residual }}$ & 5,321 & $0,047^{*}$ \\
& MOE & 1,812 & $0,242^{\mathrm{ns}}$ \\
& MOR & 0,026 & $0,975^{\mathrm{ns}}$ \\
\hline \multirow{3}{*}{ MDP } & $\varepsilon_{\text {final }}$ & 2,201 & $0,192^{\mathrm{ns}}$ \\
& $\varepsilon_{\text {residual }}$ & 3,905 & $0,082^{\mathrm{ns}}$ \\
& MOE & 4,599 & $0,062^{\mathrm{ns}}$ \\
& MOR & 3,662 & $0,091^{\mathrm{ns}}$ \\
\hline
\end{tabular}

*significativo e ns não significativo pelo teste de Tukey HSD ao nível de $\alpha=0,05$.
Sekino e Okuma (1985), estudando painéis aglomerados, observaram fadiga apenas após $10^{7}$ ciclos. A frequência utilizada no estudo foi de 1,0-2,0 Hz, com carga de 60 a $90 \%$ da resistência máxima em flexão estática. Os autores observaram que a deflexão aumenta gradualmente, até uma deformação crítica, a qual era seguida rapidamente pela ruptura da amostra. Bao e Eckelman (1995), estudando o tempo para ocorrer a fadiga em compostos de madeira usados para móveis, observaram que esse tempo decresceu com o aumento da tensão aplicada. Com uma tensão de $40 \%$ do MOR, a duração do ensaio de fadiga para o MDF foi de 290.000 ciclos, enquanto que para o MDP a duração média foi de 540.830 ciclos, a uma tensão de $50 \%$ do MOR.

Na Figura 3, apresentam-se os resultados das deformações dos painéis MDF e MDP em relação ao número de ciclos aplicados. Embora o nível de tensão aplicado tenha sido abaixo do limite elástico, foi observada, para ambos os tipos de painéis, deformação permanente $\left(\varepsilon_{\text {residual }}\right)$. Esse resultado decorre do comportamento reológico do material, o qual é observado tanto para madeira sólida como para os compostos de madeira, já que estes, assim como qualquer outro material, não apresentam, na teoria, comportamento perfeitamente elástico (BODIG; JAYNE, 1993). Laufenberg et al. (1999) e Wood (1951) destacam ainda, que essa deformação ocorre mesmo com baixos níveis de tensão e, se mantido o carregamento, esta aumentará ao longo do tempo, tendendo a provocar a ruptura do material.

No caso do MDF, o maior valor de deformação final foi obtido para 40.000 ciclos, entretanto não houve diferença estatística significativa entre os diferentes ciclos. Analisando a deformação residual, o maior valor observado foi para 80.000 ciclos, no entanto, a diferença foi estatisticamente significativa apenas, em relação aos 20.000 ciclos. Para o MDP, não foi constatada diferença significativa entre os valores de deformação final e residual (Tabela 2).

No geral, as deformações observadas para os painéis MDF foram superiores (cerca de $75 \%$ ) àquelas observadas para os MDP (Figura 3). Esses resultados estão de acordo com o observado por Pritchard et al. (2001), que estudaram variação entre deformações de diferentes variedades de painéis e verificaram que painéis OSB foram mais resistentes à fluência, quando comparados a MDF e aglomerados, sendo destes o MDF o menos resistente e o que apresentou para as tensões aplicadas, um comportamento acima do limite elástico.

Cerne, Lavras, v. 17, n. 3, p. 403-409, jul./set. 2011 


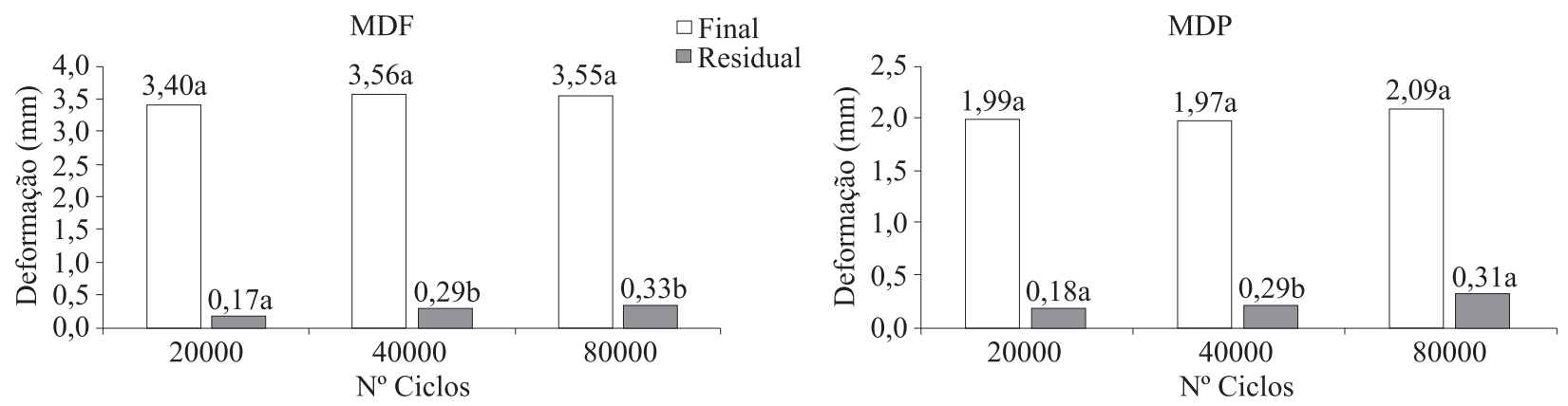

Nota: Letras iguais para uma mesma classe de deformação (final ou residual), indicam a não existência de diferença estatisticamente significativa segundo teste de Tukey HSD ao nível de $\alpha=0,05$.

Figura 3 - Deformação final e residual para os painéis MDF e MDF após carregamento cíclico.

Figure 3 - Final and residual deformation for MDF and MDP panels after cyclic loading.

Os valores médios das propriedades de flexão dos painéis MDF e MDP e os valores encontrados por Pazetto (2009), considerados como 0 ciclos, são apresentados na Figura 4. Observa-se que não houve diferença estatisticamente significativa para os valores de MOE e MOR entre os diferentes ciclos tanto para o MDF, como para o MDP (Tabela 2). Supõe-se que a não observação de diferença significativa entre o número de ciclos, tenha ocorrido por terem sido o número de ciclos e a carga (até 80.000 ciclos; 25\% MOR), relativamente baixos. Ademais, observa-se que os valores das propriedades de flexão dos painéis deste estudo são muito próximos aos valores obtidos por Pazetto (2009) para esse mesmo material sem ação da fadiga (0 ciclos). Loferski (1997), destaca que o tempo durante o qual a carga age num corpo de madeira ou produto à base de madeira, seja contínuo ou intermitente, é importante para determinar a carga que ele possa suportar.
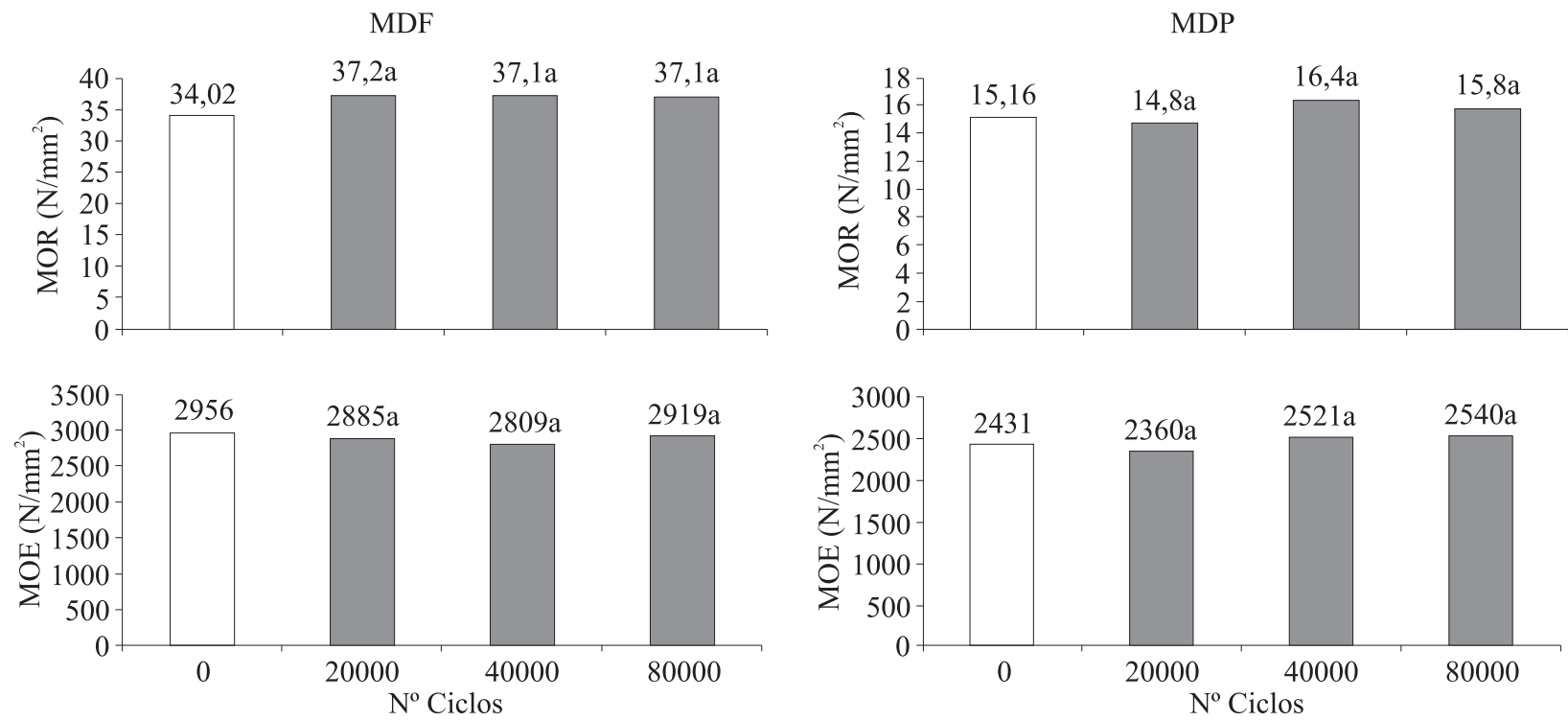

Nota: $\mathrm{N}^{\mathrm{o}}$. ciclos igual a 0 corresponde aos valores obtidos por Pazetto (2009) para o mesmo material; letras iguais indicam a não existência de diferença estatisticamente significativa segundo teste de Tukey HSD ao nível de $\alpha=0,05$.

Figura 4 - Valores médios das propriedades de flexão dos painéis MDF e MDP.

Figure 4-Average value of MOR for MDF and MDP panels.

Cerne, Lavras, v. 17, n. 3, p. 403-409, jul./set. 2011 
Em uma análise conjunta das Figuras 3 e 4, observase que embora os painéis MDF tenham apresentado as maiores deformações quando comparados ao MDP, foram eles que obtiveram os maiores módulos de resistência e rigidez. Isso ocorre porque os efeitos dependentes do tempo são complexos e inter-relacionados com inúmeras outras características físicas, mecânicas e de composição (anatômicas). Segundo Frihart (2005), quando a madeira submetida a carregamento cíclico ou contínuo, inicialmente, são causadas alterações na organização celular dos polímeros. Entretanto, nos compostos lignocelulósicos (painéis), componentes adicionais como adesivos e demais aditivos utilizados em sua manufatura, também podem influenciar nas propriedades dependentes do tempo desses produtos.

\section{CONCLUSÕES}

O número de ciclos utilizado $(20.000,40.000$ e 80.000 ciclos), bem como a intensidade de carga aplicada (25\% do módulo de ruptura) não foram suficientes para provocar fadiga e alterar os valores médios de MOE e MOR dos painéis MDF e MDP, mas provocou uma deformação residual estatisticamente significativa nos painéis MDF. Isso significa que móveis ou outros acessórios confeccionados com esses tipos de painéis, quando submetidos a carregamento prolongados podem ter sua capacidade de suportar carga reduzida.

Uma vez que o presente trabalho é um estudo preliminar, novos estudos são necessários para se avaliar a influência de diferentes intensidades de carga e um maior número de ciclos nas propriedades de flexão estática de painéis reconstituídos de madeira.

\section{AGRADECIMENTOS}

Os autores expressam seus sinceros agradecimentos ao Laboratório de Produtos Florestais (LPF), do Serviço Florestal Brasileiro, e ao Laboratório de Ensaios de Móveis (LabMov), da Universidade de Brasília, pela contribuição para a realização deste trabalho.

\section{REFERÊNCIAS}

AMERICAN SOCIETY FOR TESTING AND MATERIALS. ASTM D 1037: standard methods of evaluating the properties of wood-base fiber and particle panel materials. West Conshohocken, 1999. $30 \mathrm{p}$.

BAO, Z.; ECKELMAN, C. Fatigue life and design stresses for wood composites used in furniture. Forest Products Journal, Madison, v. 45, n. 7/8, p. 59-63, 1995.

Cerne, Lavras, v. 17, n. 3, p. 403-409, jul./set. 2011
BAO, Z.; ECKELMAN, C.; GIBSON, H. Fatigue strength and allowable design stresses for some wood composites used in furniture. Holz als Roh-und Werkstoff, Berlin, v. 54, n. 6, p. 377-382, 1996.

BODIG, J.; JAYNE, B. A. Mechanics of wood and wood composites. Davis: V. Nostrand, 1993. 712 p.

ESPINOSA, M. M.; CALIL JUNIOR, C. Statistical fatigue experiment design in medium density fiberboard. Materials Research, New York, v. 3, n. 3, p. 84-91, 2000.

FRIHART, C. R. Wood adhesion and adhesives. In: ROWELL, R. Wood chemistry and wood composites. New York: CRC, 2005.

LAUFENBERG, T. L.; PALKA, L. C.; MCNATT, J. D. Creep and creep-rupture behavior of wood-based structural panels. Madison: USDA, 1999. 574 p.

LOFERSKI, J. R. Long term performance and durability of engineered wood products. In: SMULKI, S. (Ed.).

Engineered wood products: a guide for specifiers, designers and users. Madison: PFS Research Foundation, 1997. p. 193222.

MELO, R. R.; DEL MENEZZI, C. H. S. Comportamento reológico da madeira e derivados. Ciência da Madeira, Pelotas, v. 1, n. 1, p. 25-45, 2010.

PAZETTO, V. M. F. Efeito da espessura e do vão sobre deformação em flexão de painéis de madeira utilizados na produção de móveis. 2009. 70 p. Dissertação (Mestrado em Ciências Florestais) - Universidade de Brasília, Brasília, 2009.

PRITCHARD, J.; ANSELL, M. P.; THOMPSON, R. J. H.; BONFIELD, P. W. Effect of two relative humidity environments on the performance properties of MDF, OSB and Chipboard. Wood Science and Technology, New York, v. 35, n. 5, p. 395-403, 2001.

SEKINO, N.; OKUMA, M. Performance over time of construction particleboard 1: fatigue behaviour in bending. Mokuzai Gakkaishi, Tokyo, v. 31, n. 10, p. 801-806, 1985.

SMITH, I.; LANDIS, E.; GONG, M. Fracture and fatigue in wood. Chichester: J. Wiley, 2003. 234 p. 
SUGIMOTO, T.; YAMASAKI, M.; SASAKI, Y. Fatigue and hysteresis effects in wood-based panels under cyclic shear load through thickness. Wood and Fiber Science, Madison, v. 38 , n. 2, p. 215-228, 2006.

THOMPSON, R. J. H.; ANSELL, M. P.; BONFIELD, P. W.; DINWOODIE, J. M. Fatigue in wood-based panels: part 2, property changes during fatigue cycling of OSB, chipboard and MDF. Wood Science and Technology, Madison, v. 39, n. 4, p. 311-325, 2005.

WOOD, L. W. Relation of strength of wood to duration of load. Madison: USDA, 1951. 10 p. (Technical Report, 1916).

Cerne, Lavras, v. 17, n. 3, p. 403-409, jul./set. 2011 